The Chinese Society of Theoretical and Applied Mechanics

Chinese Journal of Mechanics Press, Beijing, China

Allerton Press, INC., New York, U.S.A.

\title{
ON THE MECHANISM OF THE FORMATION OF HORIZONTAL CRACKS IN A VERTICAL COLUMN OF SATURATED SAND*
}

\author{
Che-Min Cheng (郑哲敏) ${ }^{+,++}$Tan Qingming (谈庆明) ${ }^{++}$Peng Fujiao (彭福骄) ${ }^{++}$ \\ $\left({ }^{+}\right.$State Key Laboratory of Nonlinear Mechanics, ${ }^{++}$Institute of Mechanics, Chinese Academy of \\ Sciences, Beijing 100080, China)
}

\begin{abstract}
Extended horizontal cracks have been observed experimentally in a vertical column of saturated sand when a flow of water is forced to percolate upward through it. This paper provides a theory for this phenomenon. It will be shown that the presence of inhomogeneity in permeability along the length of the column is essential for such cracks to develop. It will also be shown that small initial inhomogeneity may be magnified through the transport of the finer component of the sand by percolation. Under certain conditions liquefaction takes place at a section of the sand column causing a crack to initiate and grow there. This theory is found to be in good qualitative agreement with the experimental findings.
\end{abstract}

KEY WORDS: liquefaction, cracks, percolation

\section{INTRODUCTION}

In two recent papers experimental observations on the formation of extended horizontal cracks in vertical columns of saturated sand contained in circular cylinders have been reported ${ }^{[1,2]}$. In [1] the cylinder was subjected to an axial impact. In [2] a steady flow of water was driven upward through the column of sand sitting on a perforated rigid diaphragm. In both cases care was taken in preparing the sample by feeding wetted uniform sand continuously into a column of water to avoid intentional stratification. However, small inhomogeneity still existed.

In the literature the existence of "water film" in sand bed containing an impermeable layer was first suggested by Seed ${ }^{[3]}$ in attempting to explain slope failures observed in earthquakes. Later, Fiegel and Kutter ${ }^{[4]}$ performed a centrifuge shake table test to demonstrate the formation of water films in layered sand. More recently, Kobusho ${ }^{[5]}$ performed shake table tests using sand samples containing a seam of nonplastic silt about $4 \mathrm{~mm}$ thick and showed that a water film was formed underneath the silt layer. In this case the column was subjected to horizontal shocks simulating earthquakes. In the present paper we shall use the term crack rather than water film as attention will be focused on the condition of growth of cracks.

Received 18 May 2000, revised 1 December 2000

* The project supported by the National Natural Science Foundation of China (19832010) 
Both papers [1] and [2] find that liquefaction was a necessary condition for crack initiation and growth. In [1] the entire column of sand was liquefied following the impact. In [2] liquefaction took place locally as the flow rate was gradually raised. They found that cracks appeared only when the range of size distribution of sand grains of the specimens was broad. They also showed that when a horizontal layer of fine sand was intentionally sandwiched in the sand column a crack would develop there. In addition, [2] showed analytically that the crack should be located just beneath the fine sand layer.

This report is a follow-up of the above two papers. It reports progress on the study of the mechanism of the formation of such cracks on the basis of [1] and [2]. It will be shown that inhomogeneity of grain size distribution along the length of the sand column is an essential precondition for cracks to initiate and grow. The transport of sand composed of the fine grain component by percolation tends to cause further stratification that aggravates this inhomogeneity. While liquefaction is a necessary condition for the formation of cracks, it is by no means the only condition or a sufficient condition. In this report we shall develop a theory based mainly on the experimental results of [2]. Application of this theory to the case discussed in [1] will be presented in a separate paper.

Figures 1 and 2 are taken from [2] where $J$ represents the measured excess pore pressure gradient (with a change in sign, in the present notation) and $Y$ is the so called liquefaction index defined as the ratio of the excess pore pressure to the effective compressive stress. $Y$ greater or equal to 1 indicates liquefaction. The set of curves in Fig. 1 describes $J$ as a function of height when the driving velocity of water is gradually increased. During the experiment this velocity was increased so slow that inertia effects were negligible.

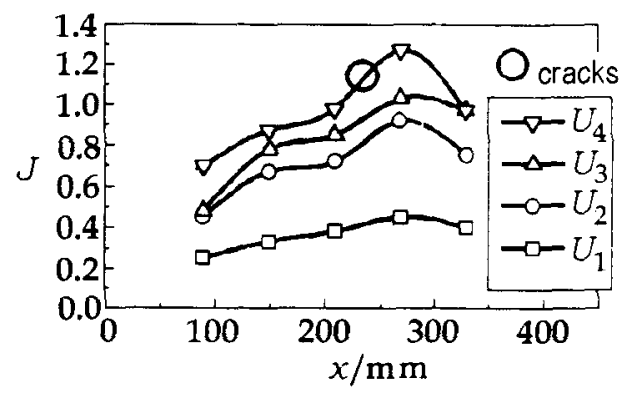

Fig.1 Pressure gradient versus $x$

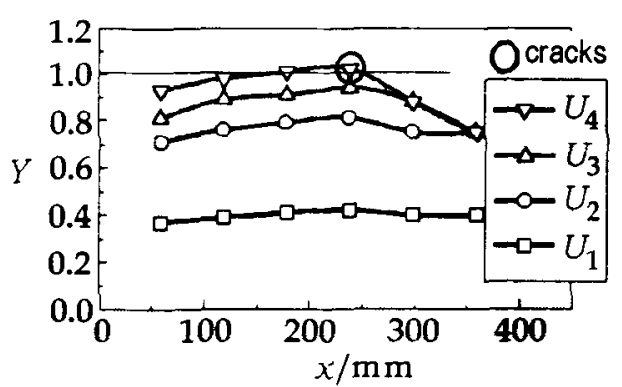

Fig.2 Liquefaction index versus $x$

$$
\left(U_{1}=0.06 \mathrm{~mm} / \mathrm{s}, U_{2}=0.11 \mathrm{~mm} / \mathrm{s}, U_{3}=0.13 \mathrm{~mm} / \mathrm{s}, U_{4}=0.17 \mathrm{~mm} / \mathrm{s}\right)
$$

The points marked by a circle in Figs. 1 and 2 designate the position where a crack formed in the experiment. We note that in Fig.2 the circled point lies on the line $Y=1$, confirming the assertion that liquefaction is a necessary condition for the formation of a crack.

\section{FORMULATION OF A THEORY ON THE INITIATION AND INITIAL GROWTH OF HORIZONTAL CRACKS}

The following simplified form of two-phase flow equations will be used. These equations are based on the assumption that the flow is one dimensional and the wall friction and inertia effect may be neglected. Only the simplest form of interaction between sand grains and water, namely Darcy's law, is taken into consideration. 


$$
\begin{aligned}
& \frac{\partial \varepsilon \rho}{\partial t}+\frac{\partial \varepsilon \rho u}{\partial x}=0 \\
& \frac{\partial(1-\varepsilon) \rho_{s}}{\partial t}+\frac{\partial(1-\varepsilon) \rho_{s} u_{s}}{\partial x}=0 \\
& \frac{\partial p}{\partial x}+\frac{\varepsilon\left(u-u_{s}\right)}{k}+\rho g=0 \\
& \frac{\partial p}{\partial x}+\frac{\partial \sigma}{\partial x}+\varepsilon \rho g+(1-\varepsilon) \rho_{s} g=0
\end{aligned}
$$

where $t$ is the time, $x$ the coordinate pointing upward with origin at the bottom of the sand column. $\varepsilon(x)$ is the porosity which, for the present, is assumed to be a known function of $x$, $\sigma$ the effective compressive stress, $u$ the pore velocity of water, $u_{s}$ the velocity of sand, $\rho$ and $\rho_{s}$ are the density of water and sand grains, respectively, $g$ the gravitational acceleration. The permeability $k(\varepsilon)$ will be regarded as a sensitive function of $\varepsilon$. For the case studied in [2] $u_{s}$ can be set to zero. The first two mass conservation equations can then be integrated to yield

$$
\varepsilon(x) u=U
$$

The integration constant $U$ obviously is the flow rate of water per unit cross sectional area of the sand column. Integrating the other two equations using the boundary conditions that $p$ and $\sigma$ are both equal to zero at $x=H$, where $H$ denotes the height of the sand column, then we have

$$
p=\rho g(H-x)+U \int_{x}^{H} \frac{\mathrm{d} x}{k(\varepsilon)} \quad \sigma=\left(\rho_{s}-\rho\right) g \int_{x}^{H}(1-\varepsilon) \mathrm{d} x-U \int_{x}^{H} \frac{\mathrm{d} x}{k(\varepsilon)}
$$

The second term in either of these equations expresses the excess pore pressure driving the percolation. The first term in the second equation represents the effective weight, the weight of the sand minus buoyancy. In the absence of wall friction the liquefaction takes place where $\sigma=0$. This is identical to the condition that the liquefaction index $Y$ defined as the ratio of the excess pore pressure over the effective weight is equal to unity. The problem of finding whether or not a crack will form is tantamount to establishing whether or not a root $x$ of the following equation exists between 0 and $H$, i.e.

$$
\frac{1}{U} F(x)=G(x)
$$

where

$$
F(x)=\left(\rho_{s}-\rho\right) g \int_{x}^{H}(1-\varepsilon) \mathrm{d} x \quad G(x)=\int_{x}^{H} \frac{\mathrm{d} x}{k(\varepsilon)}
$$

In looking for the root, an additional condition must be satisfied. This condition is imposed by the physical requirement that the sand column be sitting on the rigid diaphragm so that the effective compressive stress $\sigma$ at $x=0$ is greater than zero, i.e.

$$
F(0) / U>G(0)
$$

Since the integrands in both integrals are positive, both $F(x) / U$ and $G(x)$ are decreasing functions of $x$. They have a common intersect with the $x$ axis at $x=H$ and the intersect of $F(x) / U$ with the ordinate (Fig.3) lies above that of $G(x)$. Clearly Eq.(4) does not have a root for small $U$. 


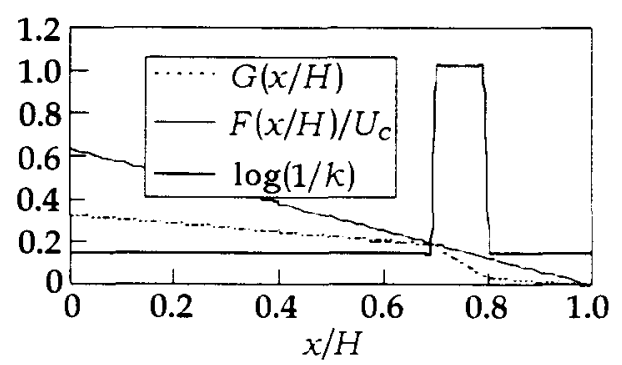

(a) Less permeable layer sandwiched

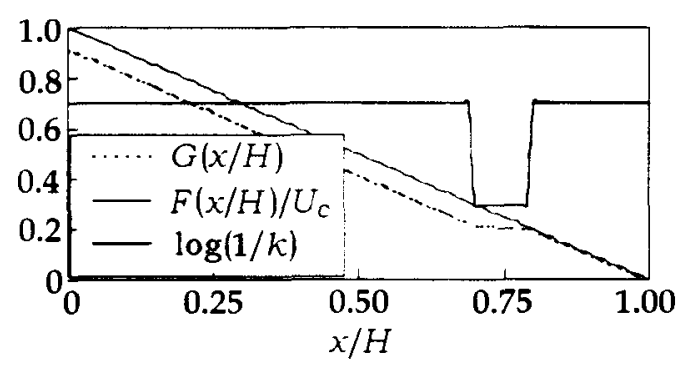

(b) More permeable layer sandwiched

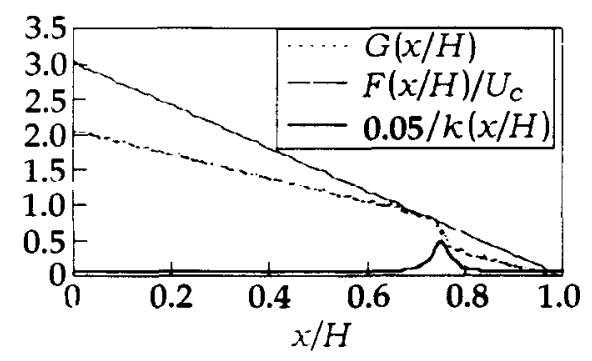

(c) Less but continuously permeable layer sandwiched

Fig.3 Liquefaction and crack development

To be more specific we assume that $\varepsilon$ is nearly equal to a constant $\varepsilon_{0}$. Then we can write

$$
F(x)=\left(\rho_{s}-\rho\right)\left(1-\varepsilon_{0}\right)(H-x) g
$$

However, as a sensitive function of $\varepsilon, k(\varepsilon)$ cannot be treated as a constant. Physically we expect $k$ to increase (or decrease) significantly for a slight increase (or decrease) in $\varepsilon$.

To look at the dynamics of crack initiation and growth in detail, let us consider cases where

$$
\begin{array}{lll}
k(\varepsilon)=k_{0} & \text { for } 0 \leq x \leq x_{1} \text { or } x_{2} \leq x \leq H \\
k=k_{0} \pm \Delta k & \text { for } x_{1} \leq x \leq x_{2} & \Delta k>0
\end{array}
$$

Figures 3(a) and 3(b) illustrate the functions $F(x) / U$ and $G(x)$ for which a layer more or less permeable is respectively sandwiched at $x / H=0.7 \sim 0.8$. It is seen that increasing $U$ simply makes the straight line representing $F(x) / U$ rotate anti-clockwise around the point $(H, 0)$. For the case of a less permeable laycr a state of liquefaction is first reached at $x / H=0.7$ as $U$ increases slowly to reach a critical value $U_{c}$, Fig.3(a). For the case of a more permeable layer the scenario is quite different. Once liquefaction takes place. the liquefaction region extends throughout the entire sand column from $x / H=0.8$ to 1. Fig.3(b).

Figure 3(c) illustrates the case for which a layer of less but continuously varying permeability is sandwiched. In this case a state of liquefaction is first reached at a cross section somewhere above $x / H=0.7$ but less than 0.8 . We note the resemblance between the theoretical curve representing the slope of $G(x)$ and the corresponding experimental curve at the points marked by a circle (Fig.1 and Fig.3(c)).

Next we address the problem of initiation and growth of a crack. Obviously liquefaction by itself does not necessarily mean crack formation. In order for a liquefied section to become a crack, a layer of water has to be formed to fill the crack. In the case of small $k$ discussed above, this requires an increase of inlet flow rate $U_{c}$ from the first appearance of liquefaction 
Vol.17, No.1 Che-Min Cheng et al.: Mechanism Study of Crack Formation in Saturated Sand 5

to a larger $U_{c}+\delta U$. The extra flow rate $\delta U$ supplies the amount of water needed to sustain the opening up of the crack. In fact the crack opening velocity is exactly equal to $\delta U$. At the same time this extra flow rate drives the column of sand above $x / H=0.7$ to move as a rigid body with velocity equal to the crack opening velocity, without altering its previous state of percolation. The relative percolation velocity in this column remains to be $U_{c}$.

\section{GROWTH OF INHOMOGENEITY ALONG THE LENGTH OF THE SAND COLUMN}

Figure 1, showing the experimentally observed excess pore pressure gradient along the length of the column measured at different flow rates, indicates that the permeability varies along the length of the column. This set of curves is characterized by two features. First, the permeability tends to decrease in a general way as $x$ increases. Second, initial inhomogeneity in permeability along $x$ persists and may even be amplified as time increases. Since crack appears where the permeability is close to its minimum, the experimentally observed inhomogeneity in permeability both in space and time is a question deserving further examination.

Experimentally when the grain size range of the sand is sufficiently narrow, cracks do not form. They form only when the grain size range is broad and contains fine grains. These observations lead to the view that the finer grains in the latter case may be flushed away by the percolating water. This will have two consequences. First, the initial porosity will be altered. Second, the percolating water becomes turbid. Both factors lead to modification of permeability in time and space. Hence the answer to our question lies in properly describing the transport of these fine grains and its effect on permeability.

Although a full description of this process is not available, a simple empirical model can be devised to explain qualitatively the main features observed experimentally.

An eroding turbid percolating flow through a porous sand can be described in terms of a three phase flow in the following manner. In addition to porosity $\varepsilon(x, t)$ we define $q(x, t)$ as the volume fraction or specific volume of sand carried in the percolating fluid. We define $Q(x, t)$ as the specific mass of sand lost to the percolating fluid. Then we have the following three mass conservation equations

$$
\begin{aligned}
& \frac{\partial(\varepsilon-q) \rho}{\partial t}+\frac{\partial(\varepsilon-q) \rho u}{\partial x}=0 \\
& \frac{\partial q \rho_{s}}{\partial t}+\frac{\partial q \rho_{s} u}{\partial x}=\frac{\partial Q}{\partial t} \\
& \frac{\partial(1-\varepsilon) \rho_{s}}{\partial t}=-\frac{\partial Q}{\partial t}
\end{aligned}
$$

Next, we simulate the erosion/deposition process using the following relations

$$
\begin{aligned}
& \frac{1}{\rho_{s}} \frac{\partial Q}{\partial t}=\frac{\lambda}{T}\left(\frac{u}{u^{*}}-q\right) \quad \text { if } \frac{Q}{\rho_{s}} \leq \frac{Q_{c}(x)}{\rho_{s}} \\
& \frac{1}{\rho_{s}} \frac{\partial Q}{\partial t} \leq 0 \quad \text { otherwise }
\end{aligned}
$$

and

$$
k=k(\varepsilon, q)
$$


where the first term on the right side of the first equation shows how the sand is being transferred to water. The second term describing deposition places a limit on the amount of sand that can be carried in the fluid. $Q_{c}(x)$ is the maximum amount of sand available that can be washed away from a unit volume element at $x$. For simplicity $Q_{c}(x)$ is assumed to be given and independent of the flow rate and the state of sand. $T$ and $u^{*}$ are empirical constants. $\lambda$ is a small parameter, employed to obtain an asymptotic solution. $k(\varepsilon, q)$, the permeability, is now assumed to be a fast varying or sensitive function of $q$ as well as $\varepsilon$

$$
k(\varepsilon, q)=k_{0} e^{-\alpha q+\beta \varepsilon} \quad 1 \ll \beta \ll \alpha
$$

We let $\beta \ll \alpha$ in order to make $k$ more sensitive to $q$ than $\varepsilon$. Equations (8) and (9) form a closed set of equations describing the kinematics of the three phase system. They will be solved subject to the following initial and boundary conditions

$$
\begin{array}{lll}
\varepsilon(x, 0)=\varepsilon_{0}(x) & q(x, 0)=0 & Q(x, 0)=0 \\
\varepsilon(0, t) u(0, t)=U(t) & q(0, t)=0 &
\end{array}
$$

The three mass conservation equations then yield

$$
\begin{gathered}
\frac{Q}{\rho_{s}}=\varepsilon(x, t)-\varepsilon_{0}(x) \\
\varepsilon(x, t) u(x, t)=U(t) \\
\frac{\partial q}{\partial t}+\frac{\partial q u}{\partial x}=\frac{\partial \varepsilon}{\partial t}
\end{gathered}
$$

The first equation in (9) becomes

$$
\frac{\partial \varepsilon}{\partial t}=\frac{\lambda}{T}\left(\frac{u}{u^{*}}-q\right)
$$

Equations (12), (13) and (14) together with the initial and boundary conditions (11) form a complete set of equations. The asymptotic solution can be obtained in the usual manner as

$$
\begin{aligned}
& u(x, t)=\frac{U(t)}{\varepsilon_{0}(x)}-\lambda \frac{U(t)}{T u^{*} \varepsilon_{0}^{3}(x)} \int_{0}^{t} U(\tau) \mathrm{d} \tau+O\left(\lambda^{2}\right) \\
& \varepsilon(x, t)=\varepsilon_{0}(x)+\lambda \frac{1}{T u^{*} \varepsilon_{0}(x)} \int_{0}^{t} U(\tau) \mathrm{d} \tau+O\left(\lambda^{2}\right) \\
& \frac{Q(x, t)}{\rho_{s}}=\lambda \frac{1}{T u^{*} \varepsilon_{0}(x)} \int_{0}^{t} U(\tau) \mathrm{d} \tau+O\left(\lambda^{2}\right)
\end{aligned}
$$

The calculation of $q$ is somewhat more complicated. The zeroth order term in $q$ is clearly equal to zero. The first order term $q_{1}(x, t)$ satisfies the following equation

$$
\frac{\partial q_{1}}{\partial t}+\frac{U(t)}{\varepsilon_{0}(x)} \frac{\partial q_{1}}{\partial x}-q_{1} \frac{U(t)}{\varepsilon_{0}^{2}(x)} \frac{\mathrm{d} \varepsilon_{0}(x)}{\mathrm{d} x}=\frac{U(t)}{T u^{*} \varepsilon_{0}(x)}
$$

of which the characteristics satisfy the following equation

$$
\frac{\mathrm{d} x}{\mathrm{~d} t}=\frac{U(t)}{\varepsilon_{0}(x)}
$$


Vol.17, No.1 Che-Min Cheng et al.: Mechanism Study of Crack Formation in Saturated Sand 7

Hence the characteristics relating $x$ and $t$ are implicitly given by

$$
\int_{x_{0}}^{x} \varepsilon_{0}(\xi) \mathrm{d} \xi=\int_{0}^{t} U(\tau) \mathrm{d} \tau \quad \int_{0}^{x} \varepsilon_{0}(\xi) \mathrm{d} \xi=\int_{t_{0}}^{t} U(\tau) \mathrm{d} \tau
$$

The first set of curves based on the parameter $x_{0}$ gives characteristics originating from the $x$ axis. The second set of curves based on the parameter $t_{0}$ gives those originating from the time axis. Integrating along a characteristic originating from the $x$ axis, $x=x\left(x_{0}, t\right)$, we obtain a formal expression for $q_{1}(x, t)$

$$
\begin{aligned}
q_{1}\left(x\left(x_{0}, t\right), t\right)= & \frac{1}{T u^{*}} \exp \left\{\int_{0}^{t} \frac{U(\tau)}{\varepsilon_{0}^{2}\left(x^{\prime}\right)} \frac{\mathrm{d} \varepsilon_{0}\left(x^{\prime}\right)}{\mathrm{d} x^{\prime}} \mathrm{d} \tau\right\} \int_{0}^{t} \frac{U(\tau)}{\varepsilon_{0}\left(x^{\prime}\right)} \\
& \cdot \exp \left\{-\int_{0}^{\tau} \frac{U\left(\tau^{\prime}\right)}{\varepsilon_{0}^{2}\left(x^{\prime \prime}\right)} \frac{\mathrm{d} \varepsilon_{0}\left(x^{\prime \prime}\right)}{\mathrm{d} x^{\prime \prime}} \mathrm{d} \tau^{\prime}\right\} \mathrm{d} \tau
\end{aligned}
$$

In this expression $x=x\left(x_{0}, t\right)$ is the characteristic originating from $x_{0}$ between 0 and $H$. Making use of Eq.(17), we obtain

$$
q_{1}(x)=\frac{1}{T u^{*}} \varepsilon_{0}(x) \int_{x_{0}}^{x} \frac{\mathrm{d} \zeta}{\varepsilon(\zeta)}
$$

The gradient of the excess pore pressure $p_{e}$ is given by

$$
\rho g J=-\frac{\partial p_{e}}{\partial x}=\frac{U(t)}{k(\varepsilon, q)}+\left(\rho_{s}-\rho\right) g q+\frac{\rho_{s} U(t)}{\varepsilon^{2}(x, t)} \frac{\partial \varepsilon}{\partial t}
$$

As $k$ varies with $\varepsilon$ and $q$ in an exponential manner, the first term on the right hand side of (21) dominates. Hence

$$
J \approx \frac{U(t)}{\rho g k(\varepsilon, q)}=\frac{U(t)}{\rho g k_{0}} \exp \left\{\alpha \lambda q_{1}-\beta\left[\varepsilon_{0}(x)+\lambda \varepsilon_{1}\right]\right\} \approx \frac{U(t)}{\rho g k_{0}} \exp \left[\alpha \lambda q_{1}-\beta \varepsilon_{0}(x)\right]
$$

To show how $J$ varies with $x$ and $t$ according to (21), we assume $U(t)=U_{0}$, namely a constant, and

$$
\varepsilon_{0}(\xi)=0.25(1-0.1 \cos 3 \pi \xi) \quad \xi=x / H
$$

$q_{1}(\xi)$ along characteristics can then be calculated using (20) and is shown in Fig.4 for $\xi_{0}=0,0.1, \cdots, 0.9$. The corresponding characteristics are shown in Fig.5. We find $q_{1}(\xi)$ 'at a given time in the following manner. At time $t U_{0} / H$ we draw an horizontal line in Fig.5. The intersection of this line with the $\xi_{0}=0$ characteristic locates a $\xi$ (note that $\left.\xi=\xi\left(\xi_{0}, 0\right)\right)$. These two values together determine the value of $q_{1}(\xi)$ from Fig.4. Repeating the same procedure for other val-

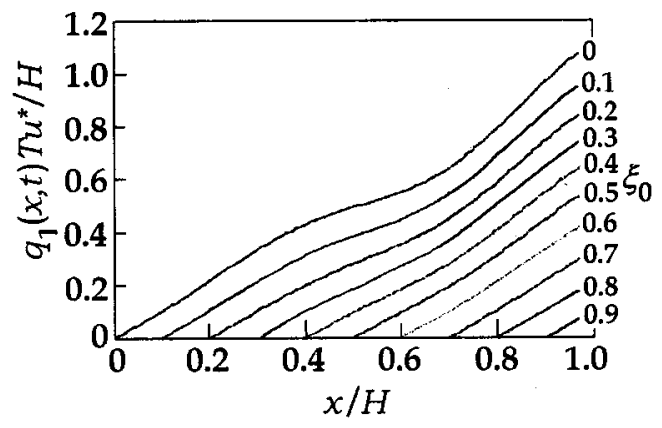

Fig.4 $q_{1}$ along characteristics ues of $\xi_{0}$ we find all values of $q_{1}(\xi)$ for this 
particular time. To obtain $q_{1}(\xi)$ for smaller values of $\xi$, we note that all characteristics originating from the time axis are constructed by simply displacing the characteristic for $\xi_{0}=0$ along the time axis (normalized to $t U_{0} / H$ in Fig.5) by appropriate amounts. Hence, the initial portion of the $q-\xi$ curve for $\xi_{0}=0$ is shared by all $q-\xi$ curves. The characteristic for $\xi_{0}=0$ reaches the top of the sand column at $t=t_{m}$ which in our numerical example is $t_{m} U_{0} / H=0.25$. For all time greater than $t_{m}$, on the first order of approximation, $\varepsilon=\varepsilon_{0}(\xi)$, and the distribution of $q$ along $x$ no longer changes with time. The first order perturbation in $q$ in the dimensionless form $T u^{*} q_{1}(x) / H$ at $t U_{0} / H=0.1$ is shown in Fig.6. It is not difficult to see that for an exact solution, our present model would yield

$$
\begin{aligned}
& \varepsilon \rightarrow \varepsilon_{0}(x)-\frac{Q_{c}(x)}{\rho_{s}} \\
& q \rightarrow 0 \quad \text { as } t \rightarrow \infty
\end{aligned}
$$

Finally, for $\alpha \lambda H / T u^{*}=0.3$ and $\beta=2$,

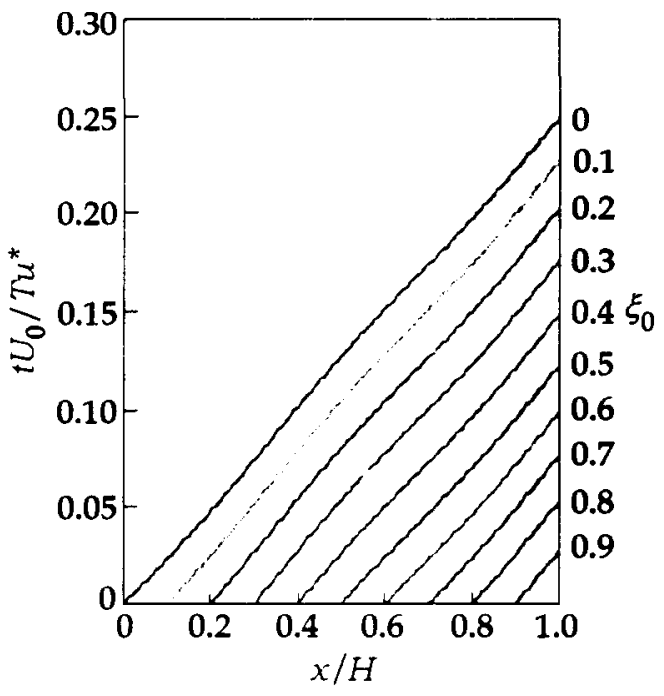

Fig.5 Characteristics $K_{0}=k_{0} \mathrm{e}^{0.5}$, the reduced excess pore pressure gradients, $\rho g J / K_{0} U_{0}$, for $t U_{0} / H=0$ and 0.25 are shown in Fig.7. Comparing this figure with Fig.1 we conclude that with appropriate choice of parameters the theoretical curves do reproduce the main features observed experimentally, namely $J$ tends to increase with some waviness as $x$ increases.

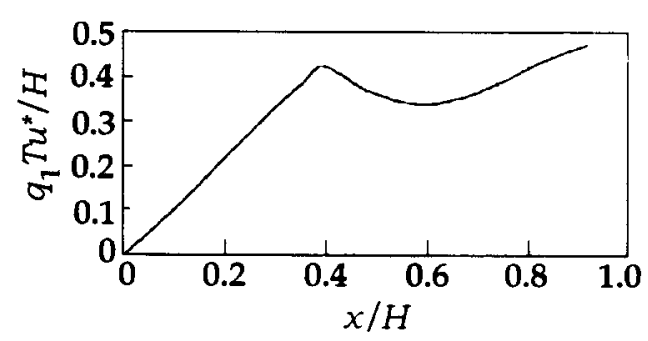

Fig.6 $q_{1}(x, t)$ at $t U_{0} / H=0.1$

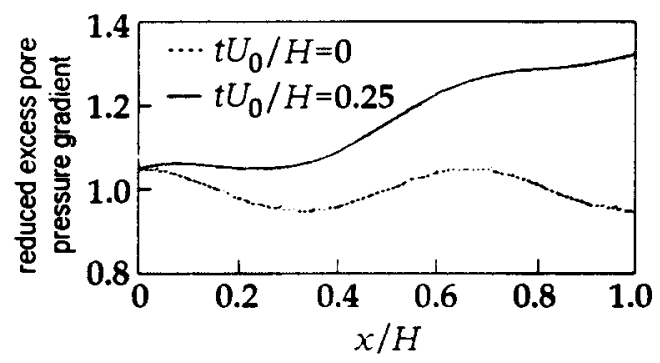

Fig.7 Reduced $J$ for initial and large $t$

Consider now the case that liquefaction first takes place at some point $\left(x_{c}, t_{c}\right)$. Since the effective stress is given by (Cf Eq.(3))

$$
\sigma(x, t)=\int_{x}^{H} g \frac{(1-\varepsilon)\left(\rho_{s}-\rho\right)(\varepsilon-q)}{\varepsilon} \mathrm{d} x-U \int_{x}^{H} \frac{\mathrm{d} x}{k(\varepsilon, q)}
$$

We have, at $x_{c}$ and $t_{c}$

$$
\int_{x_{c}, t_{c}}^{H} g \frac{(1-\varepsilon)\left(\rho_{s}-\rho\right)(\varepsilon-q)}{\varepsilon} \mathrm{d} x-U \int_{x_{c}, t_{c}}^{H} \frac{\mathrm{d} x}{k(\varepsilon, q)}=0
$$


Taking the time derivative of $\sigma$ for fixed $x$, we obtain

$$
\begin{aligned}
\left.\frac{\partial \sigma}{\partial t}\right|_{\substack{x=x_{c} \\
t=t_{c}}} & =\int_{x_{c}, t_{c}}^{H} \frac{\partial}{\partial t} g \frac{(1-\varepsilon)\left(\rho_{s}-\rho\right)(\varepsilon-q)}{\varepsilon} \mathrm{d} x+U \int_{x_{c}, t_{c}}^{H} \frac{\mathrm{d} x}{k^{2}}\left(\frac{\partial k}{\partial \varepsilon} \frac{\partial \varepsilon}{\partial t}+\frac{\partial k}{\partial q} \frac{\partial q}{\partial t}\right) \approx \\
& -\frac{\alpha \lambda U}{k_{0}} \int_{x_{c}, t_{c}}^{H} \exp \left[\alpha \lambda q_{1}(x, t)-\beta \varepsilon_{0}(x)\right] \frac{\partial q_{1}}{\partial t} \mathrm{~d} x= \\
& -\frac{\alpha \lambda U^{2} H}{T u^{*}} \int_{\xi(0, t)}^{1} \frac{\mathrm{d} \xi}{k} \frac{1}{\varepsilon_{0}(\xi)}\left(1-\frac{T u^{*}}{H} \varepsilon_{0}(\xi) \frac{\partial q_{1} / \varepsilon_{0}(\xi)}{\partial \xi}\right)
\end{aligned}
$$

for $t$ less than $t_{H}$ where $t_{H}$ is the time required for the characteristic starting from $x=0$ to reach the top of the sand column. The lower limit of the definite integral $\xi(0, t)$ is the value of $\xi$ on this characteristic at the time when a state of liquefaction prevails at $x=x_{c}$. We note that $q / \varepsilon_{0}(\xi)$ is much smaller than 1 , so that for sufficiently small value of the dimensionless parameter $T u^{*} / H$, the right hand side of (26) is always negative. This means that under such a circumstance, once a state of liquefaction is reached at a cross section of the sand column, a crack will immediately form and grow there. Unlike the case where no transport of sand is permitted, the crack grows even when the flow rate is kept constant. This is because the total force lifting the sand column above the liquefied section increases with the increase in $q_{1}$ in time.

We also note that transport of the fine component of sand tends to increase $q$ along $x$ in a general way and $q$ is the principal cause for the decrease in permeability. Now, according to (25), the smaller the permeability the closer $x_{c}$ will be to the top of the sand column. Hence the crack is more likely to be closer to the top rather than the bottom of the column. This is in full agreement with the experimental observations of both [1] and [2].

\section{CONCLUDING REMARKS}

A theory is provided in this report to explain the mechanism of the formation of extended horizontal cracks in saturated sand. It is shown that unevenness in permeability along the length of the sand column is essential for cracks to develop. Small initial unevenness in permeability or stratification can be amplified by percolation which transports the finer component of the sand through an erosion/deposition process. This process eventually brings about a condition conducive to liquefaction and crack formation. This is apparently what was observed in the experiments reported in [1] and [2].

The crude erosion/deposition model presented in (9) does appear to catch the main features of the phenomenon. To obtain more quantitative results, further work clearly needs to be done.

\section{REFERENCES}

1 Zhang JF, et al. Experimental study on permeability and settlement of saturated sand under impact load. Acta Mechanica Sinica, 1999, 31(2): 230 237 (in Chinese)

2 Peng FJ, et al. Laboratory study on cracks in saturated sands. Acta Mechanica Sinica, 2000, 16(1): 48 53

3 Seed HB. Design problems in soil liquefaction. J Geotech Engrg, ASCE, 1987, 113(8): 827 845

4 Fiegel GL, Kutter BL. Liquefaction mechanism for layered soils. J Geotech Engrg, ASCE, 1994, 120(4): 737 755

5 Kobusho T. Water film in liquefied sand and its effect on lateral spread. $J$ Geotech and Geoenviron Engrg, 1999, (Oct): 817 826 